\title{
3 Research Soure \\ miR-106b regulates the reprogramming of spermatogonial stem cells into iPSC-like cells
}

\section{Amir Hossein Hasani Fard}

Kharazmi University

Mahmoud Valizadeh

Shahid Beheshti University

\section{Zohreh Mazaheri}

Tarbiat Modares University Faculty of Medical Sciences

Jalil Hosseini ( $\nabla_{\text {jhosseinee@gmail.com ) }}$

Shahid Beheshti University of Medical Sciences https://orcid.org/0000-0003-3741-1543

\section{Research}

Keywords: MicroRNA (miRNA), Stem Cell Pluripotency, MAPK Signaling, iPSC, Bioinformatics

Posted Date: February 26th, 2021

DOI: https://doi.org/10.21203/rs.3.rs-241955/v1

License: (c) (1) This work is licensed under a Creative Commons Attribution 4.0 International License. Read Full License 


\section{Abstract}

Background Recent years have brought notable progress in raising the efficiency of the reprogramming technique, so that approaches have evolved from known transgenic factors to only a few microRNAs. Nevertheless, there is a poor understanding of both the key factors and biological networks underlying this reprogramming. Therefore, the present study aimed to investigate the potential of miR-106b in regulating Spermatogonial stem cells (SSCs) to iPSC-like cells. We used SSCs because pluripotency can be induced in them under defined culture conditions with fewer issues compared to other adult stem cells. Methods As both signaling and post-transcriptional gene control are critical for the regulation of pluripotency, we traced the expression of Oct-4, Sox-2, Klf-4, c-Myc, and Nanog (OSKMN), and studied miR-106b targets using bioinformatic methods. Results Our results showed that transfected SSCs with miR-106b increased expression of the OSKMN factors, and this expression in iPSC and induced SSC groups was significantly more than negative control groups. Moreover, using the functional miRNA enrichment analysis, online tools, and databases we predicted that miR-106b targeted a signaling pathway gene named MAPK1/ERK2, which regulates stem cell pluripotency. Conclusions Together, these data suggest that miR-106b regulates reprogramming of SSCs into iPSC-like cells by targeting the ERK2 gene as a part of the regulatory network that controls the pluripotency state and reprogramming process.

\section{Background}

Growing evidence suggests that stem cell differentiation and reprogramming techniques are quickly expanding. The discovery of induced pluripotent stem cells (iPSCs) has opened up new horizons for reprogramming technology $(1,2)$. Indeed, iPSCs and all iPSC-like cells are indispensable for generation and banking because of their aptitude to give rise to any kind of differentiated cells and tissues. They increase the potential for personalized cell therapies and introduce notable prospects for regenerative medicine, iPSC-based drug screening, disease modeling, and toxicity assessment $(3,4)$. However, somatic cells can generally be reprogrammed to iPSCs with less than $1 \%$ efficiency, as well as the clinical application of iPSCs has been laden with some issues (5). In this regard, SSCs showed can be converted into pluripotent stem cells with fewer concerns in contrast to other adult stem cells. The SSCs are postnatal germline stem cells in the testis capable of differentiating into sperm cells and can also be reprogrammed under characterized culture conditions(6, 7). Recently, SSCs have been demonstrated to be reprogrammed into multipotent SSCs (mSSCs) and pluripotency can be induced in homogeneous SSC populations without other cells. Unlike pluripotent stem cells (PSCs) with several issues, such as tumorigenicity and ethical concerns, these mSSCs can function as a pluripotent stem cell source free of the aforementioned problems $(8,9)$.

On the other hand, various substantial functions have recently emerged for microRNAs (miRNAs), singlestranded noncoding small RNAs, in the regulation of pluripotency and lineage specification $(10,11)$. miRNAs control protein synthesis by targeting mRNAs for translational repression or degradation at the posttranscriptional level. These molecules are phylogenetically conserved and have been validated to be influential in a wide assortment of core biological procedures, including embryogenesis and support of 
"stemness" among others (12). Undoubtedly, the modulation of key pluripotency factors is a critical mechanism affecting the reprogramming efficiency. Several miRNAs are discovered to be important regulators of stem cells, which modulate the expression of the transcription factors OSKMN, leading somatic cells to a pluripotent state $(13,14)$. Furthermore, the specific identification of miRNA targets will help us to understand the functional role of miRNAs in PSCs. In this field, Mei Y et al. showed the miR-21 expression pattern was highly correlated with MAPK/ERK activity during mesenchymal stem cell (MSC) differentiation $(15,16)$.

According to the features of SSCs and current studies mentioned above, we hypothesized certain miRNAs in advance as post-transcriptional regulators of SSC reprogramming. In this investigation, we selected miR-106b, a member of the miR-106b-25 cluster, because our primary bioinformatic analysis revealed that this miRNA is one of the few which affects the genes of signaling pathways regulating pluripotency of stem cells. Besides, in iPSC, the miR-106b-25 cluster is induced in early reprogramming phases and restraint of this cluster decreases the reprogramming efficiency. TGFBR2 and CDKN1A (p21) are also targets of miR-106b which have already been related to iPSC induction (17). Based on these findings, the present study was undertaken to evaluate the impact of miR-106b in cell reprogramming of SSCs to iPSClike cells by detecting the OSKMN expression. We also studied the miR-106b targets using bioinformatic methods to find out more about parts of signaling involved in the SSCs reprogramming.

\section{Methods}

\section{Animal housing}

Adult male Wistar rats, 2-2.5 months old weighing 200-300g, were used in this study $(n=6)$. These mice had free access to food and water ad libitum and were housed under a 12-h light-dark cycle at a stable temperature and humidity-controlled room.

\section{Isolation and identification of SSCs}

First of all, the testis tissue sections were excised from healthy groups, and isolation of SSCs was conducted as previously described (18). Secondly, the immunocytochemistry method was performed to anatomically visualize the localization of the promyelocytic leukemia zinc finger protein (PLZF) in the SSCs derived colonies after 7 days of culturing. The protocol of this technique was also explained earlier (18).

\section{Preparation and transfection of hsa-mir-106b plasmids}

The pLV-miRNA vector, comprising hsa-mir-106b lentivirus and co-expressing GFP protein in infected $\mathrm{E}$. coli BL21 was purchased from Biosettia Inc. (mir-p081, Biosettia, San Diego, CA, USA). Transfection of SSCs was done by $2.5 \mu \mathrm{g}$ of the pLV-miRNA vector. For this reason, mouse SSCs $\left(1.0 \times 10^{6}\right)$ were seeded 
in a 6-well plate before transfection so that the cell density was around $70 \%-90 \%$. Gently, $500 \mu$ l of culture medium and $7.5 \mu \mathrm{l}$ of Lipofectamine3000 (Invitrogen, USA) solution were mixed and incubated for 10-15 minutes at room temperature. Meanwhile, $2.5 \mu \mathrm{g}$ of the pLV-miRNA vector was added to $500 \mu \mathrm{l}$ of the medium. Then, $250 \mathrm{ml}$ of the produced solution was added to each well and the cells were incubated at $37^{\circ} \mathrm{C}$ for 2-4 days. In order to confirm the transfection efficiency and observe the expression of the GFP gene under a fluorescence microscope, the quantitative real-time reverse transcriptase PCR (qRT-PCR) technique was used. For this purpose, the sequences of forward and reverse primers, mir-106b, and U6 snRNA (as a reference gene) were downloaded from the www.ncbi.nIm.nih.gov/Gene website and designed using GeneRunner software (Table 1). The cDNA was synthesized according to the manufacturer's protocols (Fermentas, USA) and the products were analyzed by electrophoresis in $1 \%$ agarose gel with a DNA ladder (1kb).

\section{Table 1: Primers used for qRT-PCR analysis}

\begin{tabular}{|ll|}
\hline Primer & Sequence $\left(\mathbf{5}^{\prime}-\mathbf{3}^{\prime} \mathbf{)}\right.$ \\
\hline miR106b-f & ACUGCAGUGCCAGCACTT \\
miR106b-r & GGCAAAGTGCTTACAGTGC \\
\hline Stem loop & GTTGGCTCTGGTGCAGGGTCCGAGGTATTCGCACCAGAGCCAANNNNN \\
\hline $\mathrm{U}_{6}$ & AACTGGTGTCGTGGAGTC \\
\hline
\end{tabular}

\section{Preparation and culture of mouse iPSCs}

The mouse iPSC line was provided by Prof. Soleimani (Bonyakhte Stem Cells Technology, Research Center, and Tehran, Iran). iPSCs were generated from male NMRI mouse fibroblasts via the retroviral transfer of transcription factors Oct4 / Sox2 / Klf4 / c-Myc. The cells were used as positive control.

\section{Design of study and hanging drop cell culture of the experimental groups}

This study was designed based on four experimental groups, including the SSCs, SSCs with empty vector (without any miRNA gene, Mir control), SSCs infected with miR-106b (Induced SSCs), and iPSCs. We placed these experimental groups in the hanging drop culture, a simple technique that suspends media by gravity and surface tension to form 3D spheroids (19). The hanging drops were prepared by pipetting $50 \mu \mathrm{l}$ of media containing 70\% DMEM F12 (Gibco, UK), 10\% FBS (Gibco, UK), and 20\% methylcellulose (to increase the viscosity of the solution) into a $10 \mathrm{~cm}$ dish at a density of $80 \mathrm{cells} / \mu \mathrm{l}$. The bottom of the Petri dish was filled with approximately $3-4 \mathrm{ml}$ of $\mathrm{dH} 20$ to prevent drop evaporation. After that, the lid 
gently was inverted over the PBS-filled bottom chamber and incubated at $37^{\circ} \mathrm{C} / 5 \% \mathrm{CO} 2 / 95 \%$ humidity for $48 \mathrm{~h}$. The drops were monitored daily and incubated until the formation of aggregates. After $48 \mathrm{~h}$ in culture, they were transferred to a 96-well pre-coated with LM agarose. In each well, $170 \mu$ l of the $10 \%$ culture medium was then added, so that the final volume was $200 \mu$ l.

\section{Evaluation of miR-106b and OSKMN genes expression level}

The expression level of miR-106b and OSKMN genes as common pluripotent and stemness regulators were evaluated by real-time PCR. After 2 weeks of the hanging drop cell culture, the total RNA was extracted using the Trizol reagent from the experimental groups and then treated with DNase I (Fermentas, Germany) to eliminate genomic contamination. The cDNA synthesis was conducted by the RevertAid $^{\mathrm{TM}}$ First-Strand cDNA Synthesis Kit (Fermentas, Germany) and oligo (dT) primers. Thereupon, primers of the OSKMN genes and $\beta$-actin gene (as an internal control) were designed for PCR and RT reactions by Primer-BLAST tool on the NCBI database (www.ncbi.nlm.nih.gov) and synthesized via a commercial company (CinnaGen, Iran) (Table 2). The Real-Time PCR techniques were performed on Applied Biosystems, StepOne ${ }^{\mathrm{TM}}$ thermal cycler (Applied Biosystems, USA), using Master Mix and SYBR Green I. The standard PCR conditions were started by a melting cycle of $5 \mathrm{~min}$ at $95^{\circ} \mathrm{C}$ and as follows: 40 cycles of melting $\left(30 \mathrm{~s}\right.$ at $\left.95^{\circ} \mathrm{C}\right)$, annealing $\left(30 \mathrm{~s}\right.$ at $\left.60^{\circ} \mathrm{C}\right)$, and extension $\left(30 \mathrm{~s}\right.$ at $\left.72{ }^{\circ} \mathrm{C}\right)$. The melting curve analysis confirmed the quality of the reactions and then the gene efficiency (logarithmic dilution series of cDNA from the samples) was specified with a standard curve. The comparative cycle threshold (CT) method $\left(2^{-\triangle \Delta C T}\right)$ was used to examine the relative quantification of the target genes normalized against the reference gene. Then, the expressions of the target genes in studied groups were examined, compared with the gene expressions in iPSCs prior to transplantation.

Table 2: Primers used for real-time PCR analysis 


\begin{tabular}{|ll|}
\hline Primer & Sequence $\left(\mathbf{5}^{\prime} \mathbf{-} \mathbf{3}^{\prime} \mathbf{)}\right.$ \\
\hline Sox2-f & AGGGGAGAGAGAAAGAAGGAG \\
\hline Nanog-f & TATATCCACTGAGGGATCTCACCA \\
Nanog-r & CCACCTTTGGTCCCAGCATTCA \\
\hline Klf4-f & CCAACACACACGACTTCCC \\
Klf4-r & CCACGACCTTCTTCCCCTCT \\
\hline cMyc-f & TAACTCGAGGAGGAGCTGGA \\
cMyc-r & GCCAAGGTTGTGAGGTTAGG \\
\hline Oct4-f & TGATTGGCGATGTGAGTGAT \\
Oct4-r & GGAGAAGTGGGTGGAGGAAG \\
\hline$\beta$-actin-f & TCAGAGCAAGAGAGGCATCC \\
$\beta$-actin-r & GGTCATCTTCTCACGGTTGG \\
\hline
\end{tabular}

\section{Bioinformatic analysis and data availability}

First, the genes of signaling pathways regulating the pluripotency of stem cells (SPRPSCs) were downloaded from the NCBI BioSystems database (BSID: 1026136, https://www.ncbi.nlm.nih.gov/). Subsequently, the functional miRNA enrichment analysis of the genes was performed using the FunRich software (version 3.1.3) available for public access. Thereafter, we determined the potential genes which hsa-miR-106b-3p and hsa-miR-106b-5p would target by means of online tools and databases (http://www.targetscan.org/ and http://mirwalk.umm.uni-heidelberg.de/). Therefore, we generated a scalable Venn diagram to find common genes targeted by both arms of miR-106b. The common target genes and the genes of SPRPSCs ultimately were represented in a Matrix table (pair-wise comparison). Besides, we showed a Heatmap image of the common targets based on the human proteome map of the FunRich. To predict and appraise the existing protein-protein interaction (PPI) network among proteins of the common target genes distinguished in the Venn diagram, we used a bioinformatics tool, the Search Tool for the Retrieval of Interacting Genes (STRING 11.0, http://string-db.org/) database.

\section{Statistical analysis}

Gene expression analysis was performed using a one-way analysis of variance and Tukey post-test using GraphPad Prism version 8.0.0 for Windows (Graph Pad Software, San Diego, CA). Error bars represent 
$\pm S D$ (standard deviation), as well as the $p$-values $<0.05$ were considered statistically significant.

\section{Results}

\section{Characterization of SSCs}

PLZF, also known as ZBTB16 (Zinc Finger And BTB Domain Containing 16) is a consensus marker for undifferentiated spermatogonia (20). Immunocytochemistry analysis illustrated the expression of this factor in the colonies derived from the cultured cell suspensions (Fig.1).

\section{Confirmation of transfected cells}

In order to corroborate the transfection of SSCs with Mir control and the expression vector containing miR106b, the cells were observed under a Phase-contrast microscope after 48 hours. The expression of GFP protein as a reporter gene was confirmed the transfection of the cells (Fig.2).

\section{Gene expression signatures of pluripotency and miR-106b}

Real-time PCR was utilized to determine the expression of miR-106b and OSKMN, a subset of pluripotency markers, in the all group study. The findings confirmed that the SSCs remarkably were transfected with miR-106b ( $p$-value < 0.01). The mean expression of the OSKMN genes in IPSC and induced SSC groups was significantly more than negative groups. Tukey's multiple comparison test of the results verified that the iPSCs expressed the aforementioned genes more than the SSC and Mir control groups ( $p$-value $<0.01$ ). Furthermore, the difference in expression of OSKMN genes between induced SSC and iPS groups was not significant (Fig.3).

\section{miR-106b target MAPK1/ERK2 to regulate pluripotency}

The functional miRNA enrichment analysis revealed the miRNAs that target the genes of SPRPSCs (Target score: $50 \% \geq$ ). Among the miRNAs found via FunRich, miR-106b was selected based on its demonstrated potential. As the scalable Venn diagram illustrated, miR-106b targeted 7 common genes, and consequently comparing these common genes and the genes of SPRPSCs showed that miR-106b targeted ERK2 gene (Fig.4) (Table 3). In addition, the Heatmap image exhibited different expressions of ERK2 in the human proteome map, especially in the fetal and adult testis (Fig.5).

Table 3: The List of common miR-106b targeted genes 


\begin{tabular}{|lll|}
\hline Identifier & Official full name & $\begin{array}{c}\text { Mean RPKM in } \\
\text { testis }\end{array}$ \\
\hline MAPK1 & mitogen-activated protein kinase 1 & $23.469 \pm 3.817$ \\
\hline CSNK1G1 & casein kinase 1 gamma 1 & $6.568 \pm 1.596$ \\
\hline TANC2 & tetratricopeptide repeat, ankyrin repeat and coiled-coil containing & $5.949 \pm 0.57$ \\
\hline SNTB2 & syntrophin beta 2 & $5.22 \pm 0.634$ \\
\hline B4GALT6 & beta-1,4-galactosyltransferase 6 & $2.103 \pm 0.696$ \\
\hline PAK3 & p21 (RAC1) activated kinase 3 & $1.279 \pm 0.334$ \\
\hline NTNG1 & netrin G1 & $0.129 \pm 0.029$ \\
\hline
\end{tabular}

\section{Predicting PPI network of the common genes}

To gain a further understanding of the association among proteins of the common genes targeted by miR-106b, we constructed a PPI network using the STRING database. The interaction network presented no interaction between the proteins, but molecular function (GO) analysis in the database showed that PAK3 also enriched for Mitogen-Activated Protein (MAP) kinase activity (p-value: 9.50e-04) (Fig.6).

\section{Discussion}

Over the past years, much effort has been directed towards screening for small molecules to improve reprogramming efficiency and create new methods for iPSC derivation. miRNAs have played a key role in regulating pluripotency and lineage specification by modulating gene expression at the posttranscriptional level, resulting in iPSCs generation from various cell types (21-25). As a preliminary proofof-concept, the present investigation indicates that miR-106b is a major factor involved in reprogramming SSCs into iPSC-like cells. In light of the results, the SSCs infected with miR-106b expressed a subset of pluripotency markers (OSKMN) approximately in the same amount of iPSCs. In addition, the bioinformatic analysis in this study predicted that miR-106b targeted ERK2, a gene of signaling pathways that regulates stem cell pluripotency.

Transcriptional networks involving a set of pluripotent transcription factors control and sustain the pluripotency of stem cells. These pluripotent genes stimulate or suppress downstream gene expression, inducing the event of some signaling pathways and regulating the pluripotency of stem cells. As previously reported, signaling and post-transcriptional gene control are both important for pluripotency regulation, but it remains poorly known how they are incorporated to affect cell identity. In pluripotent cells, phosphorylation as a pervasive form of cell signaling plays a crucial role in controlling cell identity 
by relaying signaling of the growth factor via key pathways. The ERK2 is one of the best-characterized MAP kinase pathways that phosphorylates KIf4, OCT4, SOX2, and NANOG (26-30). For instance, The nuclear export of KLF4 requires ERK activation and the phosphorylation of KLF4 by ERK commences the interaction of KLF4 with the nuclear export factor XP01, resulting in the export of KLF4. Mutation of ERK phosphorylation site in KLF4 prevents KLF4 nuclear export, decreases in mRNA of Nanog, Klf4, and Sox2, and differentiation (31). Based on these findings and the results found in this investigation, it can be inferred that ERK2 activation controlled by miR-106b initiates reprogramming of SSCs to a pluripotent state.

Since Yamanaka and his colleagues first generated iPSCs in 2006, their clinical applications have been laden with some issues such as tumorigenicity (32). The different level of c-Myc expression that has been linked to the risk of tumorigenesis is one of the variables that hinder the therapeutic application of iPSCs. Although c-Myc alone could induce miR-106b 25 cluster, it has been reported that c-Myc activates as a proto-oncogene causing several cancers (33-36). Here, we highlight that the induced SSCs express less cMyc than iPSCs, so these iPSC-like cells have the potential to be a considerable alternative as a pluripotent stem cell source. Obviously, pathology experiments need to be conducted in the future to provide a remarkable understanding of the tumorigenicity effects of the induced SSCs.

\section{Conclusions}

Based on these findings, the SSCs can be considered as a noticeable candidate for cellular reprogramming strategies. Moreover, miR-106b was also found to be a key molecule in the SSCs reprogramming that upregulates pluripotency-associated factors. Together, our results suggest that miR$106 \mathrm{~b}$ regulates the reprogramming of SSCs into iPSC-like cells by targeting the ERK2 gene.

\section{List Of Abbreviations}

\begin{tabular}{|ll|}
\hline Abbreviation & Meaning \\
\hline SSCs & Spermatogonial stem cells \\
\hline OSKMN & Oct-4, Sox-2, KIf-4, c-Myc, and Nanog \\
\hline iPSCs & Induced pluripotent stem cells \\
\hline mSSCs & Multipotent SSCs \\
\hline PSCs & Pluripotent stem cells \\
\hline MSC & Mesenchymal stem cell \\
\hline PLZF & Promyelocytic leukemia zinc finger protein \\
\hline PPI & protein-protein interaction \\
\hline
\end{tabular}




\section{Declarations}

\section{Ethics approval and consent to participate}

All experiments were approved by the Ethics Committee of Shahid Beheshti University of Medical

Sciences and following the Declaration of Helsinki (Approval ID: IR.SBMU.REC1398.073).

\section{Consent for publication}

Not applicable.

\section{Availability of data and materials}

The datasets used and/or analysed during the current study are available from the corresponding author on reasonable request.

\section{Competing interests}

The authors declare that they have no competing interests.

\section{Funding}

This study was supported by a research grant from Men's Health \& Reproductive Health Research Center (MHRHRC), Shahid Beheshti University of Medical Sciences.

\section{Authors' contributions}

AHF and ZM and SJH proposed the work. AHF performed experimental works and data collection. MV performed bioinformatics work. MV and AHF contributed to article writing and manuscript approving. All authors read and approved the final manuscript.

\section{Acknowledgements}

This study was financially supported by Men's Health \& Reproductive Health Research Center, Shahid Beheshti University of Medical Sciences. We acknowledge the Histogenotech company and its staff who provided insight and expertise that greatly assisted the research.

\section{References}

1. Campos F. Adult stem cells and induced pluripotent stem cells for stroke treatment. Frontiers in neurology. 2019;10:908.

2. Haake K, Ackermann M, Lachmann N. Concise Review: Towards the Clinical Translation of Induced Pluripotent Stem Cell-Derived Blood Cells-Ready for Take-Off. Stem cells translational medicine. 2019;8(4):332-9. 
3. Chen K, Long Q, Xing G, Wang T, Wu Y, Li L, et al. Heterochromatin loosening by the Oct4 linker region facilitates KIf4 binding and iPSC reprogramming. The EMBO Journal. 2020;39(1):e99165.

4. Huang C-Y, Liu C-L, Ting C-Y, Chiu Y-T, Cheng Y-C, Nicholson MW, et al. Human iPSC banking: barriers and opportunities. Journal of biomedical science. 2019;26(1):87.

5. Takahashi K. Cellular reprogramming. Cold Spring Harbor perspectives in biology. 2014;6(2):a018606.

6. Guan K, Nayernia K, Maier LS, Wagner S, Dressel R, Lee JH, et al. Pluripotency of spermatogonial stem cells from adult mouse testis. Nature. 2006;440(7088):1199-203.

7. Aponte PM. Spermatogonial stem cells: Current biotechnological advances in reproduction and regenerative medicine. World journal of stem cells. 2015;7(4):669.

8. Lee Y, Lee M, Lee S-W, Choi NY, Ham S, Lee HJ, et al. Reprogramming of spermatogonial stem cells into pluripotent stem cells in the spheroidal state. Animal Cells and Systems. 2019;23(6):392-8.

9. Jeong H-S, Bhin J, Kim HJ, Hwang D, Lee DR, Kim K-S. Transcriptional regulatory networks underlying the reprogramming of spermatogonial stem cells to multipotent stem cells. Experimental \& molecular medicine. 2017;49(4):e315-e.

10. Lüningschrör $P$, Hauser $S$, Kaltschmidt $B$, Kaltschmidt $C$. MicroRNAs in pluripotency, reprogramming and cell fate induction. Biochimica et Biophysica Acta (BBA)-Molecular Cell Research. 2013;1833(8):1894-903.

11. Farzaneh M, Alishahi M, Derakhshan Z, Sarani NH, Attari F, Khoshnam SE. The expression and functional roles of miRNAs in embryonic and lineage-specific stem cells. Current stem cell research \& therapy. 2019;14(3):278-89.

12. Pourrajab F, Zarch MB, BaghiYazdi M, Hekmatimoghaddam S, Zare-Khormizi MR. MicroRNA-based system in stem cell reprogramming; differentiation/dedifferentiation. The international journal of biochemistry \& cell biology. 2014;55:318-28.

13. Ferreira AF, Calin GA, Picanço-Castro V, Kashima S, Covas DT, de Castro FA. Hematopoietic stem cells from induced pluripotent stem cells-considering the role of microRNA as a cell differentiation regulator. J Cell Sci. 2018;131(4):jcs203018.

14. Xie D, Tong M, Xia B, Feng G, Wang L, Li A, et al. Long noncoding RNA Inc-NAP sponges mmu-miR139-5p to modulate Nanog functions in mouse ESCs and embryos. RNA biology. 2020.

15. Mei Y, Bian C, Li J, Du Z, Zhou H, Yang Z, et al. miR-21 modulates the ERK-MAPK signaling pathway by regulating SPRY2 expression during human mesenchymal stem cell differentiation. Journal of cellular biochemistry. 2013;114(6):1374-84.

16. Leonardo TR, Schultheisz HL, Loring JF, Laurent LC. The functions of microRNAs in pluripotency and reprogramming. Nature cell biology. 2012;14(11):1114-21.

17. Li Z, Yang CS, Nakashima K, Rana TM. Small RNA-mediated regulation of iPS cell generation. The EMBO journal. 2011;30(5):823-34. 
18. Hasani Fard AH, Mohseni Kouchesfehani H, Jalali H. Investigation of cholestasis-related changes in characteristics of spermatogonial stem cells in testis tissue of male Wistar rats. Andrologia. 2020:e13660.

19. Velasco V, Shariati SA, Esfandyarpour R. Microtechnology-based methods for organoid models. Microsystems \& Nanoengineering. 2020;6(1):1-13.

20. Bördlein A, Scherthan H, Nelkenbrecher C, Molter T, Bösl MR, Dippold C, et al. SPOC1 (PHF13) is required for spermatogonial stem cell differentiation and sustained spermatogenesis. Journal of cell science. 2011;124(18):3137-48.

21. Lee YJ, Ramakrishna S, Chauhan H, Park WS, Hong S-H, Kim K-S. Dissecting microRNA-mediated regulation of stemness, reprogramming, and pluripotency. Cell regeneration. 2016;5(1):1-10.

22. Adlakha YK, Seth P. The expanding horizon of MicroRNAs in cellular reprogramming. Progress in Neurobiology. 2017;148:21-39.

23. Anokye-Danso F, Snitow M, Morrisey EE. How microRNAs facilitate reprogramming to pluripotency. Journal of cell science. 2012;125(18):4179-787.

24. Peskova L, Cerna K, Oppelt J, Mraz M, Barta T. Oct4-mediated reprogramming induces embryonic-like microRNA expression signatures in human fibroblasts. Scientific reports. 2019;9(1):1-13.

25. Mahabadi JA, Sabzalipoor H, Nikzad H, Seyedhosseini E, Enderami SE, Gheibi Hayat SM, et al. The role of microRNAs in embryonic stem cell and induced pluripotent stem cell differentiation in male germ cells. Journal of Cellular Physiology. 2019;234(8):12278-89.

26. Kim MO, Kim S-H, Cho Y-Y, Nadas J, Jeong C-H, Yao K, et al. ERK1 and ERK2 regulate embryonic stem cell self-renewal through phosphorylation of Klf4. Nature structural \& molecular biology. 2012;19(3):283.

27. Tsanov KM, Pearson DS, Wu Z, Han A, Triboulet R, Seligson MT, et al. LIN28 phosphorylation by MAPK/ERK couples signalling to the post-transcriptional control of pluripotency. Nature cell biology. 2017;19(1):60-7.

28. Brumbaugh J, Russell JD, Yu P, Westphall MS, Coon JJ, Thomson JA. NANOG is multiply phosphorylated and directly modified by ERK2 and CDK1 in vitro. Stem cell reports. 2014;2(1):18-25.

29. Brumbaugh J, Hou Z, Russell JD, Howden SE, Yu P, Ledvina AR, et al. Phosphorylation regulates human OCT4. Proceedings of the National Academy of Sciences. 2012;109(19):7162-8.

30. Saba-El-Leil MK, Frémin C, Meloche S. Redundancy in the world of MAP kinases: all for one. Frontiers in cell and developmental biology. 2016;4:67.

31. Dhaliwal NK, Miri K, Davidson S, El Jarkass HT, Mitchell JA. KLF4 nuclear export requires ERK activation and initiates exit from naive pluripotency. Stem cell reports. 2018;10(4):1308-23.

32. Lee AS, Tang C, Rao MS, Weissman IL, Wu JC. Tumorigenicity as a clinical hurdle for pluripotent stem cell therapies. Nature medicine. 2013;19(8):998-1004.

33. Munro MJ, Wickremesekera SK, Peng L, Marsh RW, Itinteang T, Tan ST. Cancer stem cell subpopulations in primary colon adenocarcinoma. PloS one. 2019;14(9):e0221963. 
34. Ballabio C, Anderle M, Gianesello M, Lago C, Miele E, Cardano M, et al. Modeling medulloblastoma in vivo and with human cerebellar organoids. Nature Communications. 2020;11(1):1-18.

35. Villodre ES, Felipe KB, Oyama MZ, de Oliveira FH, da Costa Lopez PL, Solari C, et al. Silencing of the transcription factors Oct4, Sox2, Klf4, c-Myc or Nanog has different effect on teratoma growth. Biochemical and biophysical research communications. 2019;517(2):324-9.

36. Mendell JT. miRiad roles for the miR-17-92 cluster in development and disease. Cell. 2008;133(2):217-22.

\section{Figures}
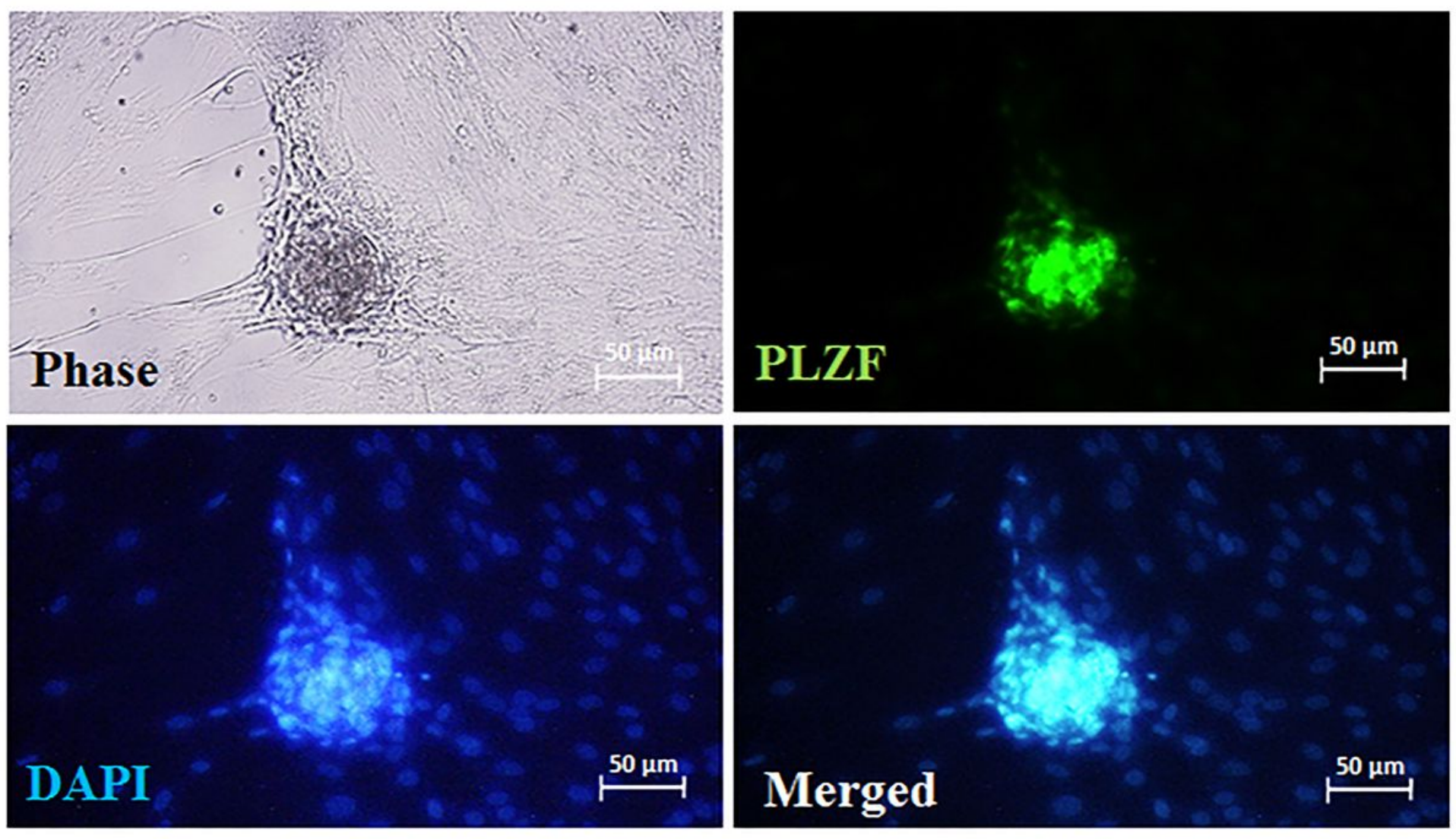

\section{Figure 1}

SSCs characterization. Immunocytochemistry revealed that PLZF protein was expressed by SSCs, as well as DAPI (chromatin marker) indicated the location of cell nuclei.

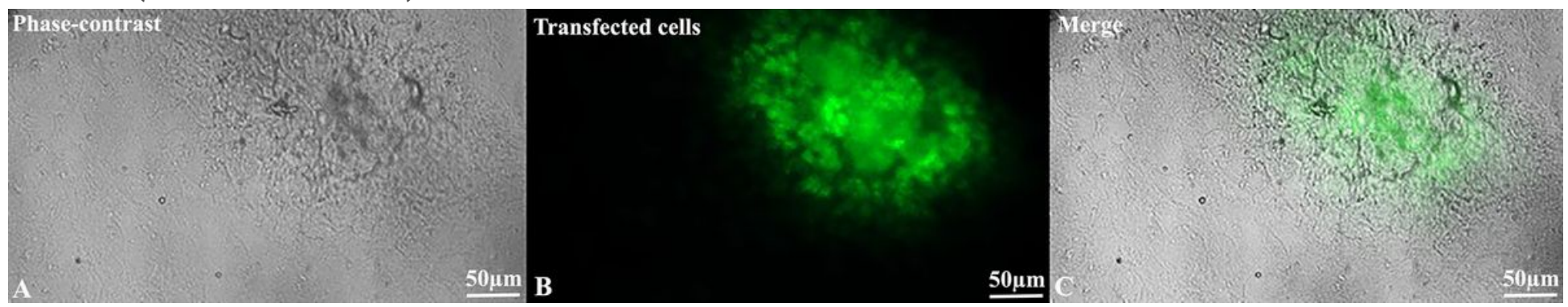


Figure 2

Evaluation of GFP protein expression after 48 hours of transfection. Images of phase-contrast (A), GFPpositive (GFP+) cells (B) and merged (C).

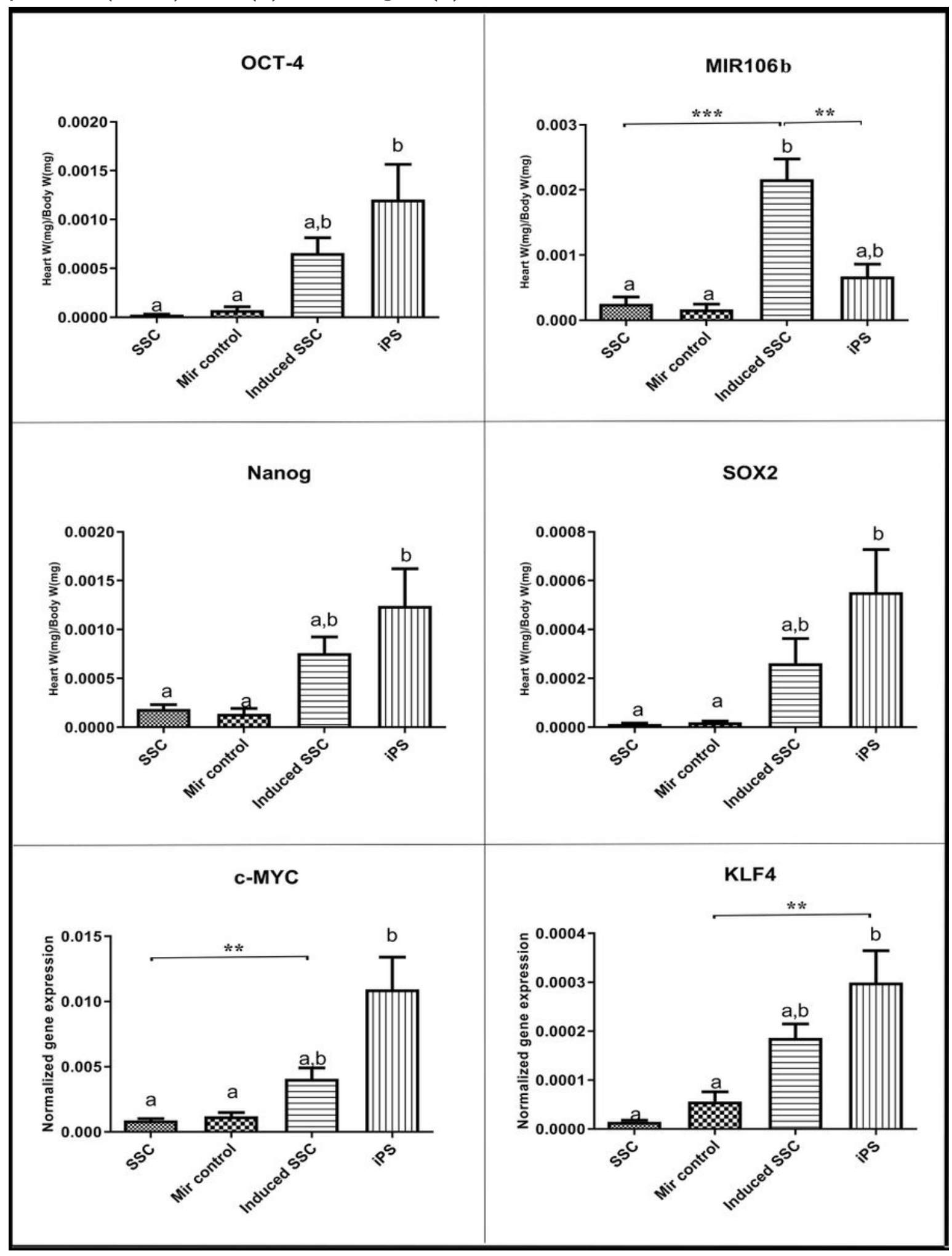

Figure 3 
Molecular assessment. Analysis of miR-106b and OSKMN expression in the experimental groups. Significant differences: $* \star \star P \leq 0.001 ; * \star P \leq 0.01$ (Error bars represent $\pm S D$ ).

A

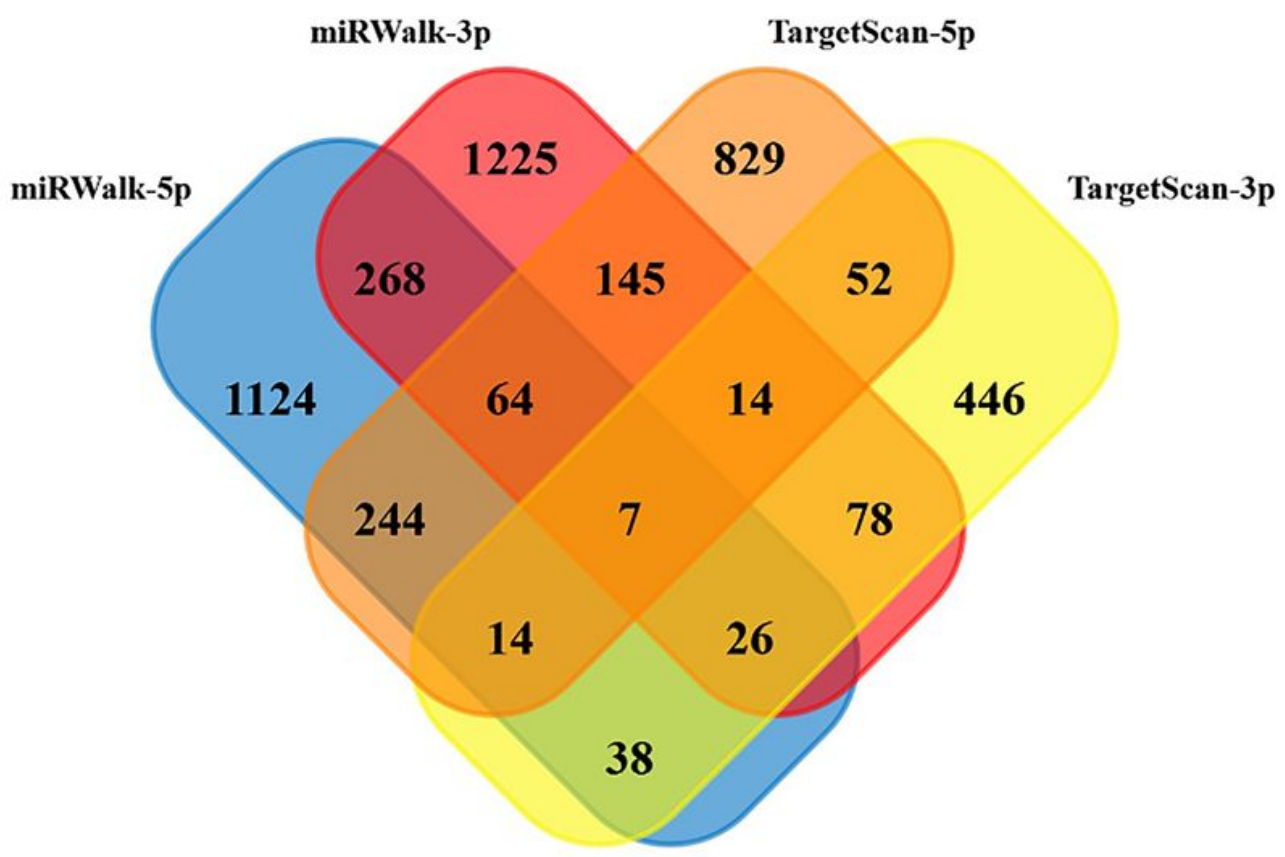

B

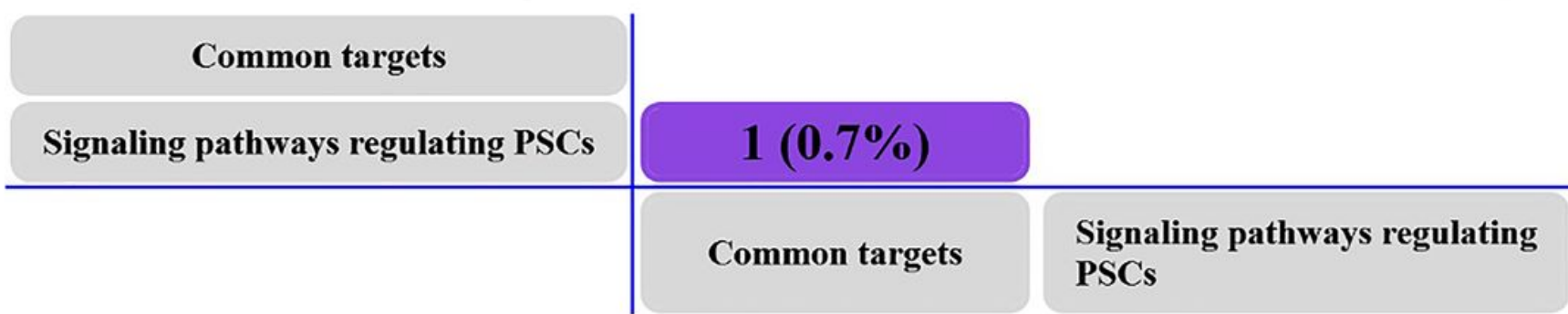

\section{Figure 4}

(A) Venn diagram showing the overlaps between the genes of miRWalk and TargetScan that miR-106b would target. (B) Matrix table (pair-wise comparison) for the common genes and the genes of SPRPSCs. 


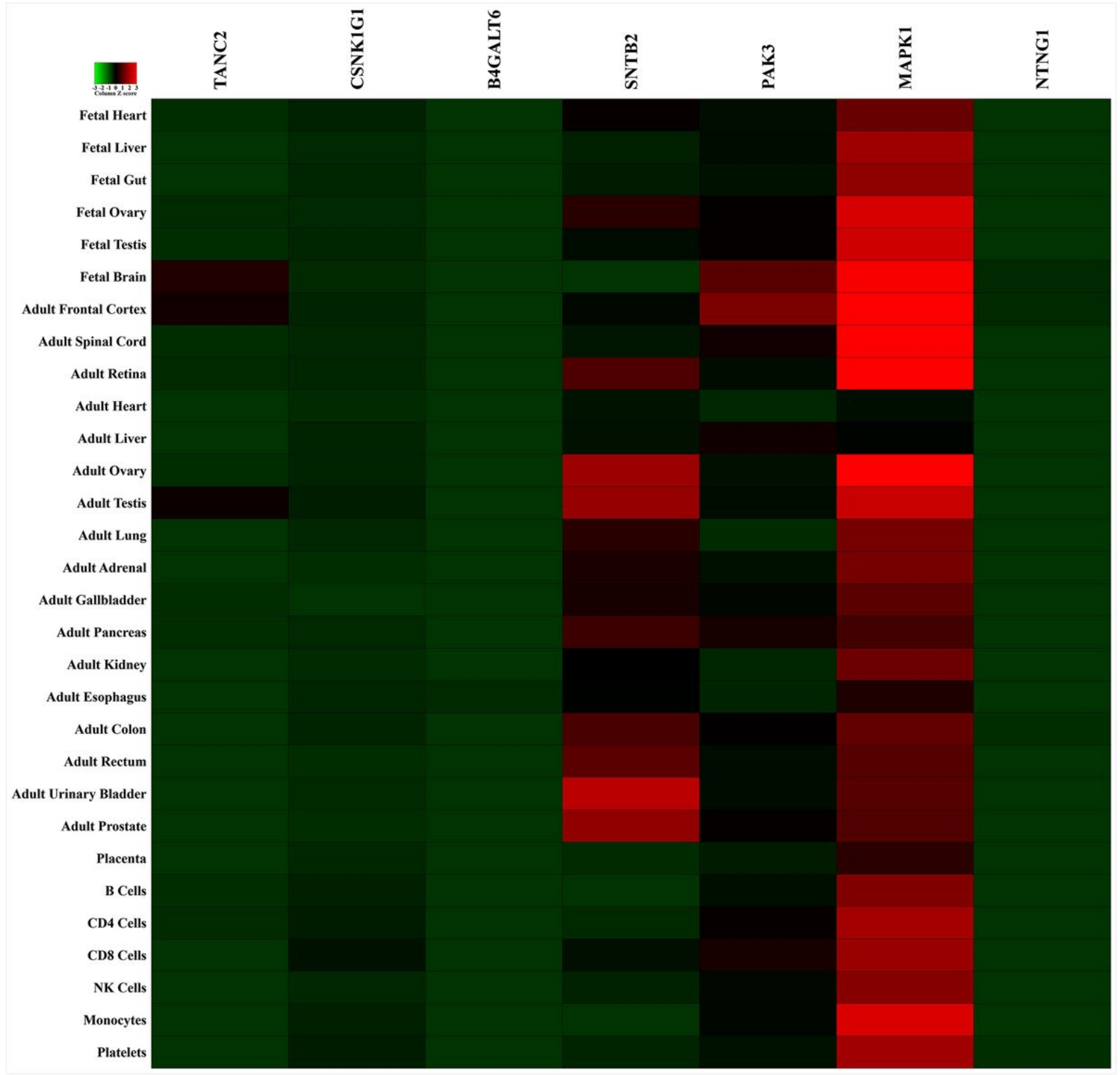

\section{Figure 5}

Heat map representing different expressions of MAPK1 (ERK2) in the human proteome map, especially in the adult and fetal testis (green-lowest abundance and red-highest abundance). 

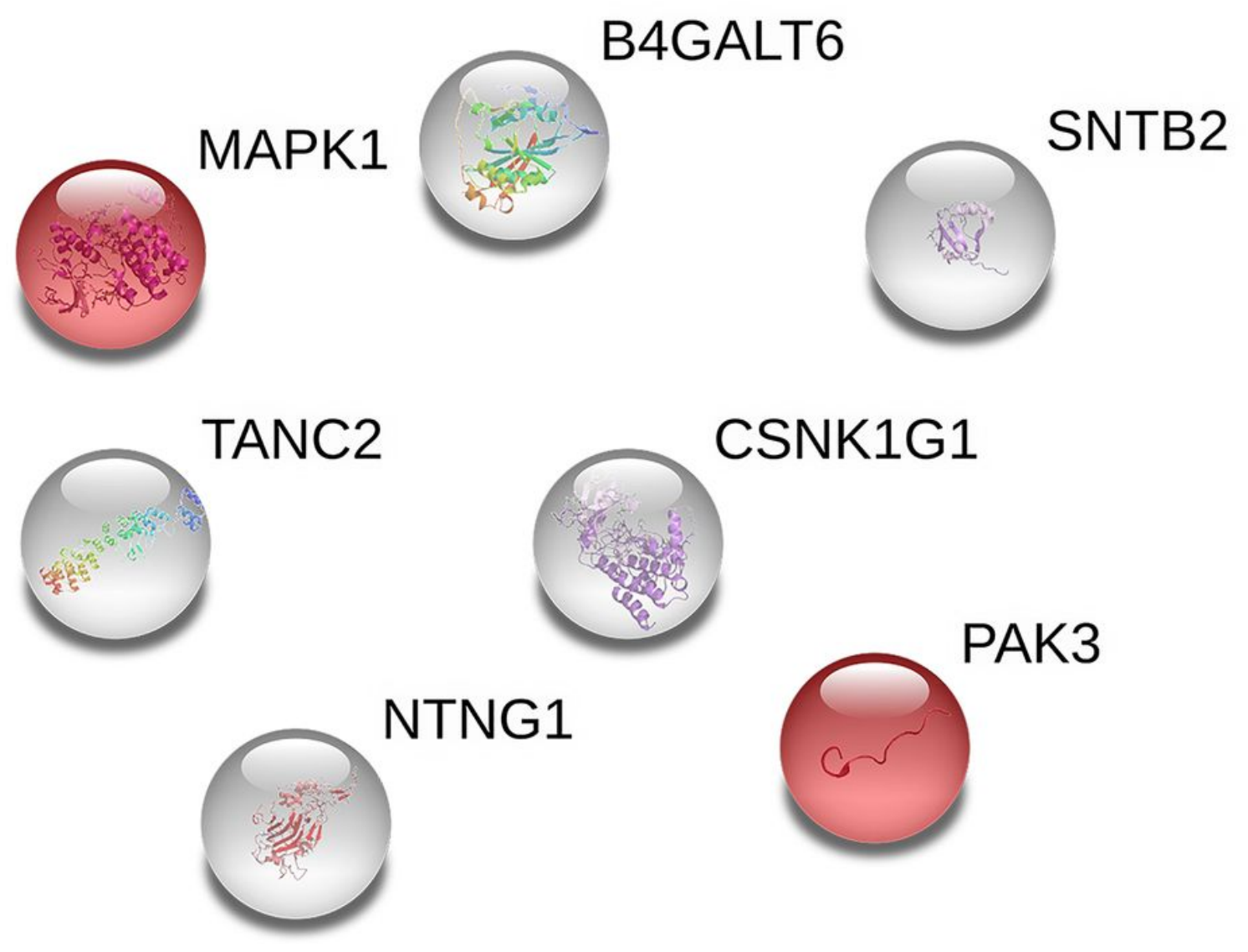

Figure 6

PPI network analysis of the common target genes using STRING database. This does not necessarily mean that they bind each other physically. 